\title{
Realizing Einstein's Mirror: Optomechanical Damping with a Thermal Photon Gas
}

\author{
A. T. M. Anishur Rahman (1) and P. F. Barker ${ }^{\dagger}$ \\ Department of Physics and Astronomy, University College London, WC1E 6BT London, United Kingdom
}

(Received 16 June 2021; accepted 26 October 2021; published 19 November 2021)

\begin{abstract}
Einstein described the damping and thermalization of the center-of-mass motion of a mirror placed inside a blackbody cavity by collisions with thermal photons. While the time for damping even a microscale or nanoscale object is so long that it is not experimentally viable, we show that this damping is feasible using the high-intensity light from an amplified thermal light source with a well-defined chemical potential. We predict this damping of the center-of-mass motion will occur on timescales of tens of seconds for small optomechanical systems.
\end{abstract}

DOI: 10.1103/PhysRevLett.127.213602

In 1909 Einstein described how an object's motion would be damped by the recoil of photons when placed inside a blackbody (BB) cavity [1]. Here, in analogy with Brownian motion, a dynamic equilibrium between the momentum fluctuations of the $\mathrm{BB}$ light and the object would bring the motional temperature of the object to that of the BB. Importantly, this was used by Einstein to understand the Planck description of BB sources [1]. This process was further explored as a potential mechanism for damping on astronomical scales [2,3] by thermal radiation from the cosmic microwave background at $3 \mathrm{~K}$. However, it was found that the damping time for any object was significantly longer than the lifetime of the Universe [2]. This damping process is weak, because as the temperature of a BB decreases, the number of photons also decreases since a BB has a chemical potential of zero.

Thermal sources of light with a well-defined chemical potential have only recently been realized [4,5]. These sources allow control over the chemical potential and therefore the number of photons for a fixed temperature. They are in a dynamic equilibrium such that the photons come into thermal equilibrium with an active medium via absorption and emission. Here, we show that when illuminated by these sources the motion of microscopic optomechanical objects are damped, while their center-of-mass motion thermalizes to the source temperature. This occurs on timescales of tens of seconds, making an experimental demonstration feasible.

Einstein initially considered the motional damping of an object by $\mathrm{BB}$ radiation when placed inside the BB cavity [1]. To illustrate this process we consider a mirror placed outside the cavity and illuminated by the light emanating from the $\mathrm{BB}$. The mirror is a disk of area $A$ and mass $M$ with a frequency dependent reflectivity $R(\omega)$. The spectral distribution of the BB photons is described by the Bose-Einstein (BE) distribution. We consider the disk in motion with velocity $v_{z}$ along the $z$ axis. Incoming photons make an angle $\theta$ with the surface normal of the disk along the $z$ axis. The number density and variance of the BB photons per unit angular frequency, solid angle, and volume are given by $\rho_{n}=\left(\omega^{2} / 4 \pi^{3} c^{3}\right)\left\{1 /\left[\exp \left(\hbar \omega / k_{B} T\right)-1\right]\right\}$ and $\Delta N^{2}=$ $\left(\omega^{2} / 4 \pi^{3} c^{3}\right)\left\{\left(\exp \left(\hbar \omega / k_{B} T\right)\right) /\left(\left[\exp \left(\hbar \omega / k_{B} T\right)-1\right]^{2}\right)\right\}$, respectively [6], where $\omega$ is the angular frequency of the photons, $k_{B}$ is the Boltzmann constant, $\hbar$ is the reduced Planck constant, $c$ is the speed of light in vacuum, and $T$ is the bulk temperature of the blackbody source. In the moving frame of the disk, the BB source appears at an effective temperature $T\left(1+\beta_{z} \cos \theta\right)^{-1}$ due to the Doppler effect $[2,7]$. The number density of photons is now given by $\rho_{n}\left(v_{z}\right)=\left(\omega^{2} / 4 \pi^{3} c^{3}\right)\left\{1 /\left(\exp \left[\hbar \omega\left(1+\beta_{z} \cos \theta\right) / k_{B} T\right]-1\right)\right\}$, where $\beta_{z}=v_{z} / c$ and $v_{z} \ll c$. The total force [2,7] delivered by all photons incident on the moving disk from a blackbody is

$$
\begin{aligned}
F_{z}\left(v_{z}\right)= & \int_{0}^{\pi / 2} \int_{0}^{2 \pi} \int_{0}^{\infty} R(\omega) c A \cos \theta \\
& \times \rho_{n}\left(v_{z}\right) 2 \hbar k \cos \theta d \omega d \Omega \\
\approx & \frac{A \pi^{2} k_{B}^{4} T^{4}}{45 c^{3} \hbar^{3}}-\frac{A \pi^{2} k_{B}^{4} T^{4}}{15 c^{4} \hbar^{3}} v_{z}
\end{aligned}
$$

where the solid angle $d \Omega$ is $\sin \theta d \phi d \theta$ and we have set $R(\omega)=1$ as considered by Einstein. The first term in Eq. (1) is the radiation pressure force, while the velocity dependent second term is the radiation damping due to recoil of photons at rate $\Gamma_{z}=\left(A \pi^{2} k_{B}^{4} T^{4} / 15 M c^{4} \hbar^{3}\right)$. The energy that the disk loses per second [6] is $M \Gamma_{z} v_{z}^{2}$, where $v_{z}^{2}=k_{B} T_{\text {c.m. }} / M$ and $T_{\text {c.m. }}$ is the center-of-mass (c.m.) temperature of the disk. In addition, due to the fluctuations in photon number $[1,6]$, the energy that the disk gains per second is

$$
\begin{aligned}
\Delta \dot{E}= & \frac{1}{2 M} \int_{0}^{\pi / 2} \int_{0}^{2 \pi} \int_{0}^{\infty} R(\omega) c A \cos \theta \\
& \times \Delta N^{2}(2 \hbar k \cos \theta)^{2} d \omega d \Omega \\
= & \frac{A \pi^{2} k_{B}^{5} T^{5}}{15 M \hbar^{3} c^{4}}
\end{aligned}
$$




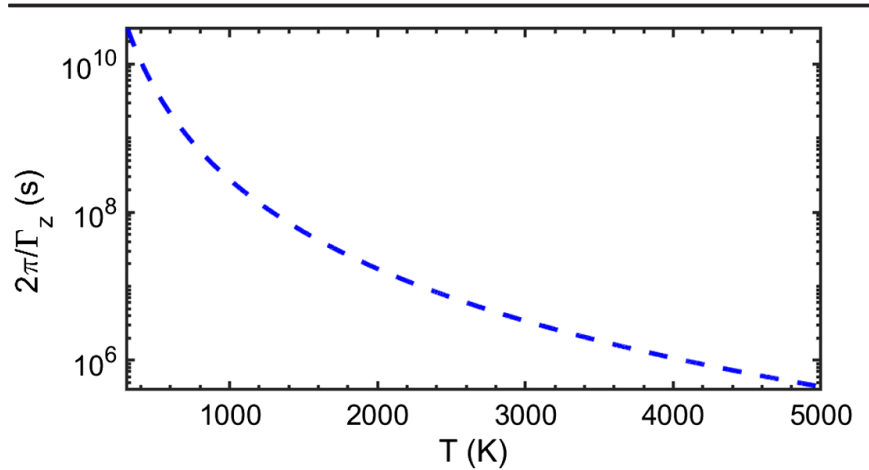

FIG. 1. The radiation damping time $2 \pi / \Gamma_{z}$ due to blackbody radiation calculated as a function of temperature, for a circular silica disk of radius $5 \mu \mathrm{m}$, thickness $50 \mathrm{~nm}$, and mass density $2200 \mathrm{~kg} \mathrm{~m}^{-3}$.

At equilibrium, the loss and the gain rate in energy are equal so that $M \Gamma_{z} v_{z}^{2}=\Delta \dot{E}$, and the c.m. temperature is equal to the BB temperature, $T_{\text {c.m. }}=T$. This is the result calculated by Einstein [1] and also later calculations for objects within the $3 \mathrm{~K}$ cosmic microwave background of the Universe [2,3].

We now consider the BB damping of a silica disk which has a radius $r=5 \mu \mathrm{m}$, thickness $50 \mathrm{~nm}$, and mass density $2200 \mathrm{~kg} \mathrm{~m}^{-3}$. The disk is placed within an ultrahigh vacuum environment $\left(\leq 10^{-9} \mathrm{mbar}\right)$ where the damping due to collisions with the residual gas molecules is negligible. The damping time $\tau_{z}=2 \pi / \Gamma_{z}$ of the mirror as a function of the BB temperature is shown in Fig. 1. The equilibration time of the mirror increases rapidly as the temperature decreases. This is due to the decrease in photon flux $\left(\propto T^{4}\right)$ with a decrease in the BB temperature [8]. At a $\mathrm{BB}$ temperature of $300 \mathrm{~K}$, the damping time for the disk is approximately 1000 years. At a temperature of $5000 \mathrm{~K}$ the damping time reduces to $\approx 4.63$ days. While this time is significantly less than that at room temperature, it is still at the limit of experimental verification [9], and producing such a high temperature BB source would be challenging.

Damping of a mirror by a $2 D$ thermal photon gas.-New thermal light sources with nonzero chemical potentials have recently been realized [4,5]. These have been produced by optical pumping of cavities containing dyes in solvents or rare earth ions within fibers. They are operated below the lasing threshold and the photons come into thermal equilibrium with the bulk temperature of the cavity. An important property of these sources is that unlike a BB source, the chemical potential and therefore the photon flux, can be controlled or even maintained when the temperature is changed $[4,10]$. This opens up the possibility of producing more intense thermal sources when compared to a BB. We show that these sources will allow the experimental realization of the optical damping of microscopic optomechanical objects $[9,11-14]$ as envisioned by Einstein.

As a concrete example, we consider a 2D microcavity filled with dye molecules in solution [see Fig. 2(a)] [4].
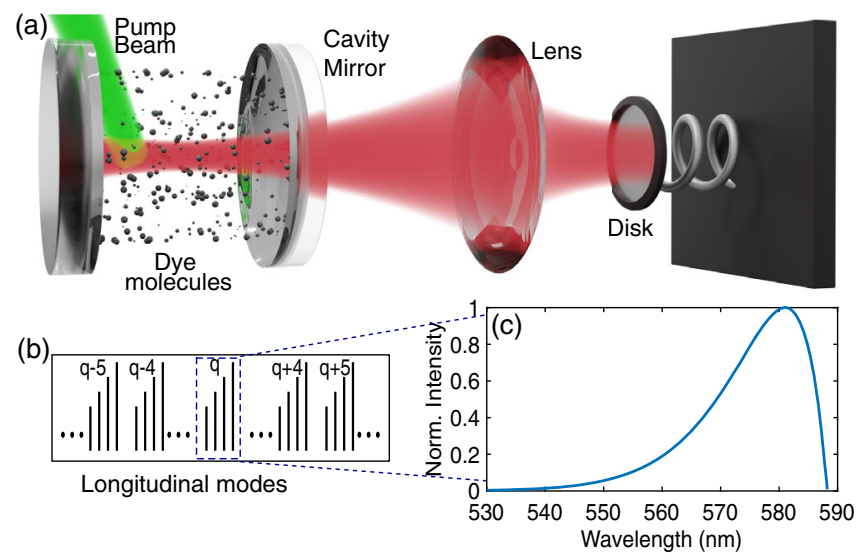

FIG. 2. A schematic diagram illustrating the damping of a mirror by thermal light. (a) A microcavity containing dye molecules in solution produces a thermal photon gas with an adjustable chemical potential. Multiple absorption-emission cycles provide the necessary thermalization of photons while the cavity traps photons facilitating thermalization and provides a well-defined set of transverse modes. Thermalization occurs in the transverse modes. The number of dye molecules and the pump power determine the chemical potential. Thermalized photons transmitted through one of the cavity mirrors are collected by a lens and focused onto a reflecting disk of reflectivity $R\left(\omega_{c}+\omega\right)=R_{0}+s\left(\omega_{c}+\omega\right)$, where $s$ is a constant. Light exiting from the cavity propagates along the $z$ axis while the disk is in the $x-y$ plane. (b) A graphical demonstration of different longitudinal modes identified by $\ldots q-5, q-4 \ldots q \ldots q+4, q+5 \ldots$ that a cavity can support. Lines within each longitudinal mode represent transverse modes. The emission spectrum of the dye molecules determines which of the longitudinal cavity modes is occupied. (c) The Bose-Einstein spectral density of a single longitudinal mode $q$, where we have assumed $2 \pi c / \omega_{c}=588.24 \mathrm{~nm}$ and $\mu_{c}=1.93 \mathrm{eV}$ [4].

The cavity traps photons emitted by the dye molecules when optically pumped. The dye molecules are a thermal bath for the photons providing the necessary chemical potential for conserving photon number when the temperature is varied [4]. The chemical potential can be adjusted by changing the number of dye molecules or the pump power [10]. The photon statistics of these sources are still given by the Bose-Einstein distribution, with the inclusion of a chemical potential $\mu_{c}$ determined by the ratio of molecules in the excited state to those in the ground state $[4,10,15]$. The energy density inside the cavity is given by

$$
\begin{aligned}
\bar{u}= & \frac{1}{V_{r}} \sum_{n_{x}=0}^{\infty} \sum_{n_{y}=0}^{\infty} 2\left(n_{x}+n_{y}+1\right) \\
& \times \frac{\hbar\left[\omega_{c}+\left(n_{x}+n_{y}+1\right) \omega\right]}{\exp \left[\left(\hbar\left[\omega_{c}+\left(n_{x}+n_{y}+1\right) \omega\right]-\mu_{c}\right) / k_{B} T\right]-1},
\end{aligned}
$$

where $n_{x}$ and $n_{y}$ are the transverse mode numbers of the cavity, $\omega=2 \pi c / n \sqrt{D_{0} R / 2}$ is the difference in frequency between two consecutive transverse modes, $\omega_{c}=q \pi c / n D_{0}$ is the angular frequency of the longitudinal cavity mode 
number $q$, and $n$ is the refractive index of the cavity filler material. The cavity mirrors have a radius of curvature $R$, with cavity volume $V_{r}$. In the limit where $\hbar \omega \ll k_{B} T$ $[10,16]$, the average number of photons transmitted through one of the cavity mirrors, per angular frequency and solid angle is $\dot{\bar{N}}=\left(V_{r} T_{r} / n q D_{0}\right)\left(\omega_{c} \omega / 4 \pi^{3} c^{2}\right) \times$ $\left.\left\{1 /\left(\exp \left[\hbar\left(\omega_{c}+\omega\right)-\mu_{c}\right) / k_{B} T\right]-1\right)\right\}$, where $T_{r}$ is the transmission coefficient of the cavity mirror (see Supplemental Material [17], Sec. iii for details). Given that $\left.\exp \left[\hbar\left(\omega_{c}+\omega\right)-\mu_{c}\right) / k_{B} T\right] \gg 1 \quad[4], \quad \dot{\bar{N}}$ can be approximated as $\left(V_{r} T_{r} / q n D_{0}\right)\left(\omega_{c} \omega / 4 \pi^{3} c^{2}\right) \times$ $\left.\left\{1 /\left(\exp \left[\hbar\left(\omega_{c}+\omega\right)-\mu_{c}\right) / k_{B} T\right]\right)\right\}$. Output powers of tens of nanowatts have been demonstrated [4,10]. Because of the relatively narrow bandwidth $(\approx 60 \mathrm{~nm}[4])$ of the light compared to a blackbody source, it can be amplified using optical amplifiers with gain $G$. We assume that the photon statistics are not significantly modified by the amplification process by noting that an optical amplifier, in addition to replicating the photon statistics of the input, adds a small thermal field typically via amplified spontaneous emission $[18,19]$. The addition of a thermal component to an already thermal source will not change the input statistics. This has more recently been shown to hold for broadband amplification of the thermal light produced by amplified spontaneous emission [20]. Amplifiers do not, however, have a flat spectral profile. This can be ameliorated by using a spectral filter on the input to compensate for this variation $[11,21,22]$. When illuminated with this amplified light, the force on the mirror in the moving frame is

$$
\begin{aligned}
F_{z}= & \frac{G V_{r} T_{r} \exp \left[\mu_{c} / k_{B} T\right]}{q n D_{0}} \\
& \times \int_{0}^{\frac{\pi}{2}} \int_{0}^{2 \pi} \int_{0}^{\infty} \frac{\omega_{c} \omega}{4 \pi^{3} c^{3}} \frac{R(\omega) 2 \hbar\left(\omega_{c}+\omega\right) \cos ^{2} \theta d \omega d \Omega}{\exp \left[\left(\hbar\left(\omega_{c}+\omega\right)\right)(1+\beta \cos \theta) / k_{B} T\right]} \\
\approx & \frac{2 G}{3 c} P-\frac{G}{2 c^{2}} \frac{\hbar \omega_{c}}{k_{B} T} P v_{z},
\end{aligned}
$$

where $\quad P=\exp \left[\left(\mu_{c}-\hbar \omega_{c}\right) / k_{B} T\right]\left(V_{r} T_{r} k_{B}^{2} T^{2} \omega_{c}^{2} /\right.$ $\left.2 q n D_{0} \pi^{2} \hbar c^{2}\right)$ is the cavity output power before amplification. The diameter of the incident light beam [see Fig. 2(a)] is equal to or smaller than that of the disk. Since the 2D thermal light source is spectrally narrow compared to a BB source, disks can have $100 \%$ reflectivities in this spectral range such that $R(\omega)=1$. The damping rate of the disk is $\Gamma_{z}=\left(G / 2 M c^{2}\right)\left(\hbar \omega_{c} / k_{B} T\right) P$. The rate of energy gain due to the fluctuation in photon number is now $\Delta \dot{E}_{z} \approx\left(G \hbar \omega_{c} / 2 M c^{2}\right) P$. The equilibrium center-of-mass temperature of the mirror is $T_{\text {c.m. }}=$ $\Delta \dot{E}_{z} / k_{B} \Gamma_{z}=T$ which is the same as that obtained for the BB source. In this case, however, both $\Gamma_{z}$ and $\Delta \dot{E}_{z}$ are adjustable through the chemical potential $\mu_{c}$ and the optical gain $G$. Figure 3 shows the damping time $2 \pi / \Gamma_{z}$ at $T=300 \mathrm{~K}$ as a function of the normalized chemical

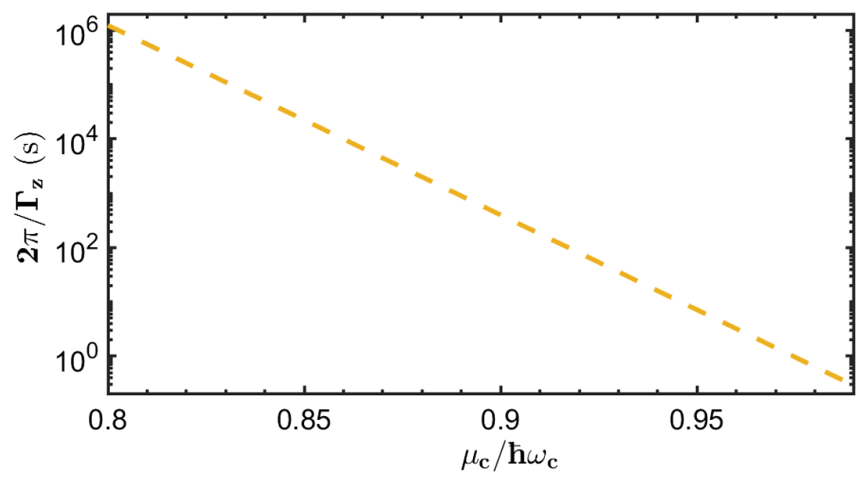

FIG. 3. The relaxation time, $2 \pi / \Gamma_{z}$, of a $5 \mu \mathrm{m}$ radius and $50 \mathrm{~nm}$ thick silica disk due to a thermal light source as a function of the normalized chemical potential. The cavity parameters [4] are: $T_{r}=1.5 \times 10^{-5}, q=7, D_{0}=1.46 \mu \mathrm{m}, \quad n=1.43 \quad$ (ethylene glycol), and $R=1 \mathrm{~m}$. The thermal source is at $T=300 \mathrm{~K}$ with an optical gain $G=80 \mathrm{~dB}$.

potential $\mu_{c} / \hbar \omega_{c}$. The parameters used in our calculation are typical of 2D experimental microcavities [4]. For a chemical potential $\mu_{c} / \hbar \omega_{c}=0.92$, and an optical amplifier gain of $80 \mathrm{~dB}$, we calculate a damping time of $2 \pi / \Gamma_{z} \approx 80 \mathrm{sec}$. This is eight orders of magnitude less than a BB source at the same temperature. If the mirror is not perfectly reflecting, the damping time will increase but importantly the equilibrium CM temperature remains the same and has been shown to hold for a linear change in the reflectivity (see Supplemental Material [17]).

A levitated dielectric sphere damped by a thermal photon gas. - We consider a levitated dielectric sphere of radius $r \ll 2 \pi c / \omega_{c}$ with a scattering cross section of $\sigma_{s}=\left(\alpha^{2} \omega_{\text {in }}^{4} / 6 \pi \epsilon_{0}^{2} c^{4}\right)$ illuminated by amplified 2D thermal light from a microcavity. The frequency of the incident light is $\omega_{\text {in }}=\omega_{c}+\omega$ and $\alpha$ is the polarizability of the particle [23]. Furthermore, we assume that the amplified light is tightly focused using a lens to a spot size of area $A_{w}$. The particle could be levitated in a Paul trap [9], or by the thermal light itself [11]. In the laboratory frame, the wave vector of an incident photon is given by $\mathbf{k}_{\text {in }}=\left[k_{x} k_{y} k_{z}\right]$, where $k_{\text {in }}=\omega_{\text {in }} / c$. In the particle frame, the incident and scattered photon frequency is $\omega_{\text {in }}(1+\beta)$, where $\beta=\left(\mathbf{v} \cdot \mathbf{k}_{\text {in }} / c k_{\text {in }}\right)$, and $\mathbf{v}=\left[v_{x} v_{y} v_{z}\right]$ is the velocity of the particle along the three axes. The unpolarized incident photons means that the scattered photons are isotropically distributed over $4 \pi$ steradians. After a scattering event, each photon delivers momentum to the particle equivalent to $\mathbf{p}=\hbar k_{\text {in }}(1+\beta)\left(\boldsymbol{\Theta}_{\text {in }}-\boldsymbol{\Theta}_{s}\right)$, where $\boldsymbol{\Theta}_{\text {in }}=\left[\sin \theta_{\text {in }} \cos \phi_{\text {in }} \sin \theta_{\text {in }} \sin \phi_{\text {in }} \cos \theta_{\text {in }}\right]$ and $\boldsymbol{\Theta}_{s}=\left[\sin \theta_{s} \cos \phi_{s} \sin \theta_{s} \sin \phi_{s} \cos \theta_{s}\right]$. Here, $\theta_{\text {in }}$ and $\phi_{\text {in }}$ represents the polar and the azimuthal angles that the wave vector of an incoming photon makes with the $-z$ axis and the $+x$ axis respectively. $\theta_{s}$ and $\phi_{s}$ are the corresponding angles that the wave vector of a scattered photon makes with the same reference axes. With the appropriate Lorentz transformation, the total force exerted by all photons is 

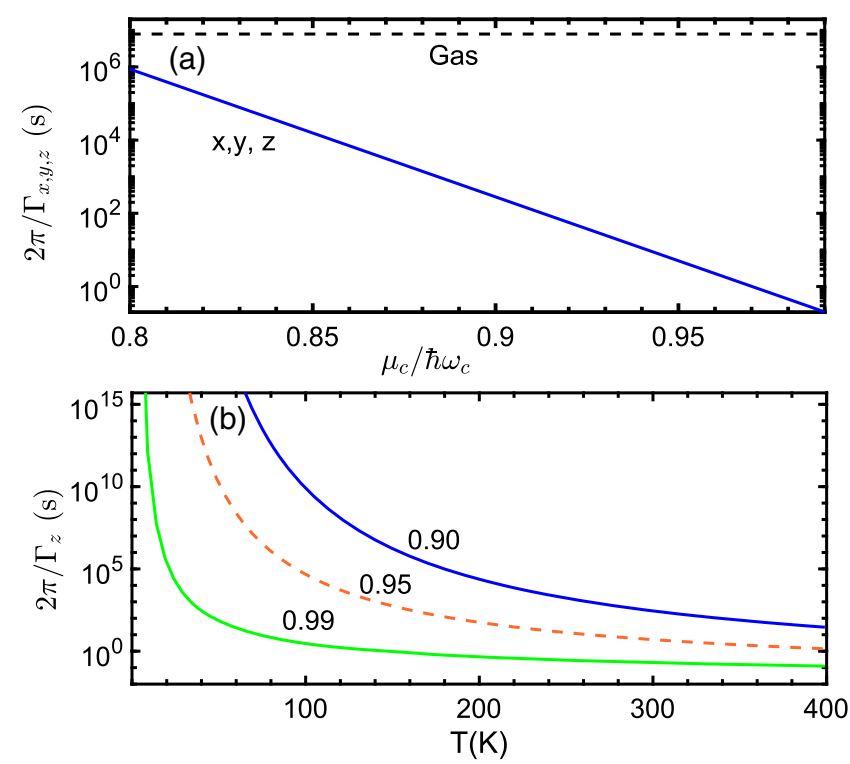

FIG. 4. (a) The damping time $2 \pi / \Gamma_{x, y, z}$ of a $200 \mathrm{~nm}$ radius silica sphere along the $x, y$, and $z$ axes when the particle is illuminated with a thermal photon gas focused to a spot size of $w=1 \mu \mathrm{m}$. The relaxation time is calculated for the light produced by a $2 \mathrm{D}$ cavity and amplified by $G=70 \mathrm{~dB}$ [4]. At $\mu_{c} / \hbar \omega_{c}=0.92$, the equivalent optical power after amplification is $\approx 200 \mathrm{~mW}$. For comparison, we have also included the equilibration time through the collisions with the residual gas molecules. In this case, the background gas pressure is $1 \times 10^{-9} \mathrm{mBar}$. (b) The relaxation time of the sphere from part (a) along the $z$ axis as a function of temperature for three different chemical potentials.

$$
\begin{aligned}
\mathbf{F}= & \frac{G V_{r} T_{r}}{q n D_{0}} \frac{1}{4 \pi} \int_{0}^{\pi} \int_{0}^{2 \pi} \int_{0}^{\frac{\pi}{2}} \int_{0}^{2 \pi} \int_{0}^{\infty}\left[\frac{\sigma_{s}}{A_{w}} \frac{\omega_{c} \omega}{4 \pi^{3} c^{2}}\right. \\
& \left.\times \frac{\exp \left(\mu_{c} / k_{B} T\right)}{\exp \left[\hbar \omega_{\text {in }}(1+\beta) / k_{B} T\right]} \hbar k_{\text {in }}\left(\boldsymbol{\Theta}_{\text {in }}-\boldsymbol{\Theta}_{s}\right)\right] d \omega d \Omega_{\text {in }} d \Omega_{s} \\
\approx & -\frac{G}{3 c^{2}} \frac{\hbar \omega_{c}}{k_{B} T} \frac{P \sigma_{c}}{A_{w}} \mathbf{v}
\end{aligned}
$$

where $\sigma_{c}=\left(\alpha^{2} \omega_{c}^{4} / 6 \pi \epsilon_{0}^{2} c^{4}\right), d \Omega_{\text {in }}=\sin \theta_{\text {in }} d \phi_{\text {in }} d \theta_{\text {in }}$, and $d \Omega_{s}=\sin \theta_{s} d \phi_{s} d \theta_{s}$. We have only shown the velocity dependent term (see Supplemental Material [17], Sec. v). The damping rate along the three axes is given by $\Gamma_{x, y, z}=\left(G / 3 M c^{2}\right)\left(\hbar \omega_{c} / k_{B} T\right)\left(P \sigma_{c} / A_{w}\right)$. Figure 4(a) shows the time required by a $200 \mathrm{~nm}$ radius silica sphere to reach equilibrium. Because of the isotropic nature of scattering, the particle requires the same time to reach the equilibrium along all three axes. For $\mu_{c} / \hbar \omega_{c}=0.92$, the required time for the particle to reach equilibrium is $2 \pi / \Gamma_{x, y, z} \approx 60 \mathrm{sec}$. Figure 4(b) shows the effect of the cavity bulk temperature on the thermalization time. For a fixed chemical potential, the damping time increases rapidly as the temperature decreases. However, this can be counteracted by increasing the chemical potential $[4,10]$.

The increase in the kinetic energy of the particle due to the fluctuation in the photon number $[1,24,25]$ is

$$
\begin{aligned}
\Delta \dot{E}= & \frac{1}{2 M} \frac{G V_{r} T_{r}}{q n D_{0}} \frac{1}{4 \pi} \int_{0}^{\pi} \int_{0}^{2 \pi} \int_{0}^{\frac{\pi}{2}} \int_{0}^{2 \pi} \int_{0}^{\infty}\left[\frac{\sigma_{s}}{A_{w}} \frac{\omega_{c} \omega}{4 \pi^{3} c^{2}}\right. \\
& \left.\times \frac{\exp \left(\mu_{c} / k_{B} T\right)}{\exp \left(\hbar \omega_{\text {in }} / k_{B} T\right)} \hbar^{2} k_{\text {in }}^{2}\left(\boldsymbol{\Theta}_{\text {in }}-\boldsymbol{\Theta}_{s}\right)^{2}\right] d \omega d \Omega_{\text {in }} d \Omega_{s} \\
\approx & \frac{\Lambda G}{3 M c^{2}} \hbar \omega_{c} \frac{\sigma_{c} P}{A_{w}}
\end{aligned}
$$

where $\boldsymbol{\Lambda}=\left[\begin{array}{lll}1 & 1 & 1\end{array}\right]$. The equilibrium center-of-mass temperature of the particle along all three axes is equal to the bulk temperature $T$ of the dye molecules, i.e., $T_{\text {c.m. }}=T$. This is strikingly different to the value calculated for a laser trapped spherical particle in high-vacuum [26] where $T_{\text {c.m. }}=$ $\hbar \omega_{l} / 4 k_{B}=6115 \mathrm{~K}$ for a $\lambda_{l}=2 \pi c / \omega_{l}=588 \mathrm{~nm}$ laser.

For a $200 \mathrm{~nm}$ radius sphere in UHV, it takes $\approx 7.90 \times$ $10^{6} \mathrm{sec}$ to reach equilibrium via the collisions with residual gas molecules. This is 5 orders of magnitude larger than when the sphere is illuminated with the thermalized photon gas. For an actual measurement of the radiation damping encountered by a levitated object, the object can be heated (cooled) to a higher (lower) energy state, for example, by rapidly manipulating the trapping potential [14,27], followed by a ring-down (reheating) measurement for determining $\Gamma$.

Finally, as the mirror and the levitated dielectric sphere are harmonically bound and oscillate at frequency $\omega_{q}$, their motion in UHV can be described by a Langevin equation $\left(d^{2} q / d t^{2}\right)+\Gamma_{q}(d q / d t)+\omega_{q}^{2} q=f_{q}(t) / M$, where $q=x, y, z$ and $f_{q}(t)$ is the random force due to the thermal photons with an autocorrelation magnitude of $2 M \Gamma_{q} k_{B} T$. Experimentally, the damping rate $\Gamma_{q}$ can be determined from the power spectral density [28] calculated from a record of the displacement as a function of time. The c.m. temperature can be found from $\int_{0}^{\infty} S_{q q}(d \omega / 2 \pi)=$ $\left(k_{B} T_{\text {c.m. }} / 2 M \omega_{q}^{2}\right)$. The damping rates calculated for both the mirror and the levitated nanosphere above have linewidths in the $10 \mathrm{mHz}$ range which are well within experimental reach [9].

We have shown that small, well isolated optomechanical systems can be damped and thermalized by a thermal photon gas as originally envisioned by Einstein. Such an experiment is feasible using thermal light sources $[4,5]$ and recent advances in optomechanics [11,13,14]. An experimental demonstration using a levitated optomechanical object, such as a charged nanoparticle in a Paul trap [9] or a neutral nanoparticle in an optical trap [12,13,29,30], in ultrahigh vacuum seems ideal. Although we have calculated the damping from a 2D source, a 1D source [5] could potentially be focused more tightly, leading to a higher damping. Amplified LEDs and superluminescent diodes, which produce thermal light [8,31] and have been used to trap dielectric spheres [11], could also be considered for investigating thermal radiation damping. Our results raise the possibility that by increasing or decreasing the bulk temperature of a thermalized light source one 
can heat or cool the center-of-mass temperature of a levitated nanoparticle without requiring feedback cooling $[11,13,27,29,30]$. Lastly, although we have considered damping by a thermal light source with an adjustable chemical potential, it would also be interesting to study the interaction of an optomechanical object with a photon BEC $[10,32,33]$, where $\mu_{c}=1$.

The authors acknowledge funding from the EPSRC Grants No. EP/S000267/1 and EP/N031105/1 as well as the H2020-EU.1.2.1 TEQ project Grant agreement ID: 766900 .

*a.rahman@ucl.ac.uk

†p.barker@ucl.ac.uk

[1] A. Einstein, On the present status of the radiation problem, Phys. Z. 10, 185 (1909), https://einsteinpapers.press .princeton.edu/vol2-doc/577.

[2] C. V. Heer and R. H. Kohl, Theory for the measurement of the earth's velocity through the $3 \mathrm{~K}$ cosmic radiation, Phys. Rev. 174, 1611 (1968).

[3] G. R. Henry, R. B. Feduniak, J. E. Silver, and M. A. Peterson, Distribution of blackbody cavity radiation in a moving frame of reference, Phys. Rev. 176, 1451 (1968).

[4] J. Klaers, F. Vewinger, and M. Weitz, Thermalization of a two-dimensional photonic gas in a white wall photon box, Nat. Phys. 6, 512 (2010).

[5] R. Weill, A. Bekker, B. Levit, M. Zhurahov, and B. Fischer, Thermalization of one-dimensional photon gas and thermal lasers in erbium-doped fibers, Opt. Express 25, 18963 (2017).

[6] M. Mansuripur and P. Han, Thermodynamics of radiation pressure and photon momentum, in Optical Trapping and Optical Micromanipulation XIV, edited by K. Dholakia and G. C. Spalding (International Society for Optics and Photonics, SPIE, Bellingham, Washington, D.C. 2017), Vol. 10347, pp. 196-215.

[7] P. J. E. Peebles and D. T. Wilkinson, Comment on the anisotropy of the primeval fireball, Phys. Rev. 174, 2168 (1968).

[8] P. Wurfel, The chemical potential of radiation, J. Phys. C 15, 3967 (1982).

[9] A. Pontin, N. P. Bullier, M. Toroš, and P. F. Barker, Ultranarrow-linewidth levitated nano-oscillator for testing dissipative wave-function collapse, Phys. Rev. Research 2, 023349 (2020).

[10] J. Klaers, The thermalization, condensation and flickering of photons, J. Phys. B 47, 243001 (2014).

[11] A. T. M. A. Rahman and P.F. Barker, Optical levitation using broadband light, Optica 7, 906 (2020).

[12] A. T. M. A. Rahman, A. C. Frangeskou, P. F. Barker, and G. W. Morley, An analytical model for the detection of levitated nanoparticles in optomechanics, Rev. Sci. Instrum. 89, 023109 (2018).

[13] J. Vovrosh, M. Rashid, D. Hempston, J. Bateman, M. Paternostro, and H. Ulbricht, Parametric feedback cooling of levitated optomechanics in a parabolic mirror trap, J. Opt. Soc. Am. B 34, 1421 (2017).
[14] V. Jain, J. Gieseler, C. Moritz, C. Dellago, R. Quidant, and L. Novotny, Direct Measurement of Photon Recoil from a Levitated Nanoparticle, Phys. Rev. Lett. 116, 243601 (2016).

[15] D. N. Sob'yanin, Bose-Einstein condensation of light: General theory, Phys. Rev. E 88, 022132 (2013).

[16] E. E. Müller, General theory of Bose-Einstein condensation applied to an ideal quantum gas of photons in an optical microcavity, Phys. Rev. A 100, 053837 (2019).

[17] See Supplemental Material at http://link.aps.org/ supplemental/10.1103/PhysRevLett.127.213602 for a detailed derivation.

[18] J. P. Gordon, L. R. Walker, and W. H. Louisell, Quantum statistics of masers and attenuators, Phys. Rev. 130, 806 (1963).

[19] W. S. Wong, H. A. Haus, L. A. Jiang, P. B. Hansen, and M. Margalit, Photon statistics of amplified spontaneous emission noise in a 10-gbit/s optically preamplified directdetection receiver, Opt. Lett. 23, 1832 (1998).

[20] I. V. Doronin, E. S. Andrianov, A. A. Zyablovsky, A. A. Pukhov, Y.E. Lozovik, A. P. Vinogradov, and A. A. Lisyansky, Second-order coherence properties of amplified spontaneous emission, Opt. Express 27, 10991 (2019).

[21] M. Harurnoto, M. Shigehara, and H. Suganurna, Gain-flattening filter using long-period fiber gratings, J. Lightwave Technol. 20, 1027 (2002).

[22] C. A. Marques, R. A. Oliveira, A. A. Pohl, and R. N. Nogueira, Adjustable EDFA gain equalization filter for DWDM channels based on a single LPG excited by flexural acoustic waves, Opt. Commun. 285, 3770 (2012).

[23] C. F. Bohren and D. R. Huffman, Particles small compared with the wavelength, in Absorption and Scattering of Light by Small Particles (Wiley-VCH Verlag GmbH, New York, 2007), pp. 130-157.

[24] T. Seberson and F. Robicheaux, Distribution of laser shot-noise energy delivered to a levitated nanoparticle, Phys. Rev. A 102, 033505 (2020).

[25] W. M. Itano and D. J. Wineland, Laser cooling of ions stored in harmonic and penning traps, Phys. Rev. A 25, 35 (1982).

[26] L. Novotny, Radiation damping of a polarizable particle, Phys. Rev. A 96, 032108 (2017).

[27] F. Tebbenjohanns, M. Frimmer, A. Militaru, V. Jain, and L. Novotny, Cold Damping of an Optically Levitated Nanoparticle to Microkelvin Temperatures, Phys. Rev. Lett. 122, 223601 (2019).

[28] M. Aspelmeyer, T. J. Kippenberg, and F. Marquardt, Cavity optomechanics, Rev. Mod. Phys. 86, 1391 (2014).

[29] J. Gieseler, B. Deutsch, R. Quidant, and L. Novotny, Subkelvin Parametric Feedback Cooling of a Laser-Trapped Nanoparticle, Phys. Rev. Lett. 109, 103603 (2012).

[30] G. Ranjit, M. Cunningham, K. Casey, and A. A. Geraci, Zeptonewton force sensing with nanospheres in an optical lattice, Phys. Rev. A 93, 053801 (2016).

[31] S. Hartmann and W. Elsaber, A novel semiconductor-based, fully incoherent amplified spontaneous emission light source for ghost imaging, Sci. Rep. 7, 41866 (2017).

[32] J. Klaers, J. Schmitt, F. Vewinger, and M. Weitz, BoseEinstein condensation of photons in an optical microcavity, Nature (London) 468, 545 (2010).

[33] R. Weill, A. Bekker, B. Levit, and B. Fischer, Bose-Einstein condensation of photons in an erbium-ytterbium co-doped fiber cavity, Nat. Commun. 10, 747 (2019). 